\title{
DETERMINAÇÃO DAS CONSTANTES DE UMA EQUAÇÃO TIPO ANTOINE A PARTIR DE EXPERIMENTOS DE ELEVAÇÃO DE PONTO DE EBULIÇÃO DE SOLUÇÕES DE LEITE/SACAROSE.
}

\author{
W. G. RODRIGUES ${ }^{1}$ T. S. TOMASELLO ${ }^{1}$, T. C. POLACHINI e J. TELIS-ROMERO ${ }^{1}$ \\ ${ }^{1}$ Universidade Estadual Paulista, Departamento de Engenharia e Tecnologia de Alimentos \\ E-mail para contato: walissonguilhermitt@gmail.com
}

\begin{abstract}
RESUMO - O presente trabalho de pesquisa apresenta uma análise de dados experimentais de 11 amostras de leite semidesnatado/sacarose em diferentes concentrações de sólidos totais. A elevação do ponto de ebulição foi determinado experimentalmente usando evaporador rotativo modificado (Marconi, Brasil). Os resultados experimentais foram simulados usando modelo empírico de Antoine que mostrou um bom comportamento frente aos resultados experimentais, mostrando valores do coeficiente de correlação ajustado de 0.999 . Os resultados obtidos nesta pesquisa podem proporcionar informações úteis para a modelagem, desenho e controle das operações unitárias envolvidas na indústria de leite condensado.
\end{abstract}

\section{INTRODUÇÃO}

A produção mundial de leite em 2013 foi de 552.091.000 toneladas, o que representou um crescimento global de 7,33\% da produção de 2010. O Brasil apresentou um aumento, nesse período, correspondente a 8,12\% (USDA, 2017).

O Brasil é o maior produtor de leite da América Latina com 32.380 mil toneladas produzidas. Com essa produção, o Brasil ocupa o quinto lugar no "rank" mundial de produtores de leite, perdendo apenas para União Europeia, Índia, Estados Unidos e China (USDA, 2013). Parte da produção mundial de leite é utilizada para a preparação do leite condensado, leite em pó e leite evaporado.

Com relação ao leite condensado, em 2016, o Brasil exportou cerca de $27 \%$ do valor total exportado de derivados lácteos. Já o leite em pó, é o líder na exportação representando $55,6 \%$ do valor total (CONAB, 2016). Entretanto, o processamento de leite em pó e também de leite condensado, em escala industrial, requer algumas propriedades do leite.

Embora algumas propriedades do leite se encontram listadas na literatura como calor específico, viscosidade dinâmica, condutividade térmica e densidade (MINIM et al, 2002), dados da elevação do ponto de ebulição (EPE) do leite ainda não estão disponíveis na literatura especializada. Portanto, o conhecimento dessa propriedade desperta um grande interesse na indústria de produtos concentrados de leite para o projeto de um evaporador de 
múltiplos efeitos nesta área. Neste trabalho foi determinado o EPE de soluções de leite semidesnatado/sacarose em função da sua concentração de sólidos totais e da temperatura.

\section{METODOLOGIA}

\subsection{Material}

Para a preparação das amostras dos experimentos foi adquirido num supermercado local de São José do Rio Preto 24 litros de leite semidesnatado pasteurizados e homogeneizados e 20 quilogramas de açúcar cristal ambos materiais de um mesmo lote.

A composição do leite foi determinada pelas seguintes analises oficiais: gorduras, proteínas, água e cinzas seguindo a metodologia proposta pelo Instituto Adolfo Lutz (2008) e carboidratos por diferença. A composição do leite semidesnatado com 8 repetições encontrase na tabela 1 , juntamente com a média seguida do desvio padrão.

Tabela 1 - Composição de leite semidesnatado pasteurizado e homogeneizado.

\begin{tabular}{cccccccccccc}
\hline & \multicolumn{10}{c}{ Amostra } \\
\cline { 2 - 11 } $\begin{array}{c}\text { Componente } \\
\text { s (\%) }\end{array}$ & $\mathbf{1}$ & $\mathbf{2}$ & $\mathbf{3}$ & $\mathbf{4}$ & $\mathbf{5}$ & $\mathbf{6}$ & $\mathbf{7}$ & $\mathbf{8}$ & Media & $S_{y}$ \\
\hline Água & 89,575 & 89,583 & 88,333 & 88,913 & 88,919 & 88,726 & 89,388 & 88,983 & 89,052 & 0,437 \\
Proteína & 3,849 & 3,849 & 3,687 & 3,708 & 3,704 & 3,714 & 3,714 & 3,728 & 3,747 & 0,065 \\
Carboidrato & 3,946 & 3,944 & 5,395 & 4,770 & 4,767 & 4,959 & 4,276 & 4,680 & 4,592 & 0,505 \\
Gordura & 1,686 & 1,868 & 1,649 & 1,668 & 1,669 & 1,663 & 1,658 & 1,667 & 1,668 & 0,013 \\
Cinzas & 0,944 & 0,938 & 0,936 & 0,941 & 0,941 & 0,939 & 0,937 & 0,943 & 0,940 & 0,003 \\
\hline
\end{tabular}

\subsection{Preparação das amostras}

Para a determinação do EPE das misturas leite/sacarose foram preparadas 11 amostras com diferentes concentrações a partir de amostras iniciais com $92 \%$ em peso de leite semidesnatado e $8 \%$ de sacarose. O procedimento para a obtenção dessas concentrações foi realizado utilizando um Evaporador Rotativo a Vácuo, marca MARCONI, modelo MA120/TH, com capacidade até $2500 \mathrm{ml}$. As misturas leite sacarose foram submetidas a diferentes etapas de evaporação obtendo-se diferentes composições determinadas a partir de balanços de massa (Tabela 2).

Tabela 2 - Onze amostras de diferentes concentrações obtidas através de evaporação para se obter o EPE de leite semidesnatado.

\begin{tabular}{cccccc}
\hline Amostra & Água & Proteína & Carboidratos & Gordura & Cinzas \\
\hline 1 & 81,928 & 3,447 & 12,225 & 1,535 & 0,865 \\
2 & 77,568 & 4,279 & 15,174 & 1,905 & 1,074 \\
3 & 74,279 & 4,906 & 17,399 & 2,184 & 1,231 \\
4 & 70,512 & 5,625 & 19,947 & 2,504 & 1,411 \\
5 & 66,163 & 6,455 & 22,889 & 2,873 & 1,619 \\
6 & 61,588 & 7,328 & 25,984 & 3,261 & 1,838 \\
7 & 56,726 & 8,256 & 29,273 & 3,674 & 2,071 \\
8 & 50,856 & 9,376 & 33,244 & 4,172 & 2,352 \\
9 & 45,003 & 10,493 & 37,203 & 4,669 & 2,632 \\
10 & 37,591 & 11,907 & 42,217 & 5,298 & 2,987 \\
11 & 29,622 & 13,427 & 47,608 & 5,974 & 3,368 \\
\hline
\end{tabular}




\subsection{Análise estatística}

Modelos empíricos foram estabelecidos usando o procedimento "Regression Model Selection" do programa Statgraphics ${ }^{\circledR}$ Centurion XV (Statpoint Technologies Inc., Warrenton, VA, USA), usando equação de Antoine (2) em função da concentração e temperatura. $\mathrm{O}$ ajuste do coeficiente de correlação $\left(R^{2}{ }_{a d j}\right)$ foi usado para verificar a eficiência dos modelos (Castell-Palou et al. 2012).

$$
R_{a d j}^{2}=1-\frac{S_{y x}^{2}}{S_{y}^{2}}
$$

\subsection{Experimento para determinar elevação do ponto de ebulição de misturas leite/sacarose}

A elevação do ponto de ebulição (EPE) foram determinados com 11 diferentes composições, Tabela 2, e pressão de 7,0 a 70,3 kPa. Neste trabalho o equilíbrio vapor-líquido do sistema foi obtido utilizando-se um evaporador rotativo modificado (Marconi, Brasil) com refluxo total da fase de vapor (Figura 1). Em cada experimento introduzia-se uma amostra de $500 \mathrm{~mL}$ da mistura de leite/sacarose no evaporador rotativo, o qual foi aquecido por um banho termostático à base de óleo de silicone. A pressão do sistema foi controlada e ajustada por uma bomba de vácuo (Marconi, Brasil), monitorada com um manômetro "U”, de mercúrio, de precisão de $0,1 \mathrm{mmHg}$. Utilizou-se um recipiente entre a bomba e o aparelho para evitar variações da pressão. Foi utilizado um banho de água termostático para resfriar o fluido do condensador a $283,15 \pm 0,1 \mathrm{~K}$ para aumentar a força motriz na condensação da fase de vapor. A diferença de temperatura entre o banho de óleo de silicone e a mistura leite/sacarose em ebulição foi mantida a aproximadamente $283,15 \mathrm{~K}$ para manutenção da força motriz afim que aconteça a evaporação. A mistura leite-sacarose foi mantida em processo evaporativo até o equilíbrio ser atingido (constante $\mathrm{T}$ e $\mathrm{P}$ ). A temperatura da mistura leite/sacarose foi medida com um sensor de precisão PT-100 calibrado $( \pm 0,1 \mathrm{~K})$ e a concentração da fase líquida $\left({ }^{\circ} \mathrm{Brix}\right.$ a $293,15 \mathrm{~K}$ ) foi medida usando um medidor de densidade digital calibrado (DMA 4500 BRASEQ, ausustria) para verificar a concentração durante o experimento.

Figura 1 - Sistema usado para medir elevação do ponto de ebulição: (A) banho termostático de água, (B) coletor de amostra, (C) sensor PT-100 sensor, (D) condensador, (E) banho de óleo de silicone, $(\mathrm{F})$ evaporador rotatório, $(\mathrm{G})$ controlador de temperatura, $(\mathrm{H})$ Erlenmeyer para evitar variações de pressão, (J) Erlenmeyer de seguridade, (K) bomba de vácuo, (M) manômetro de mercúrio tipo U.

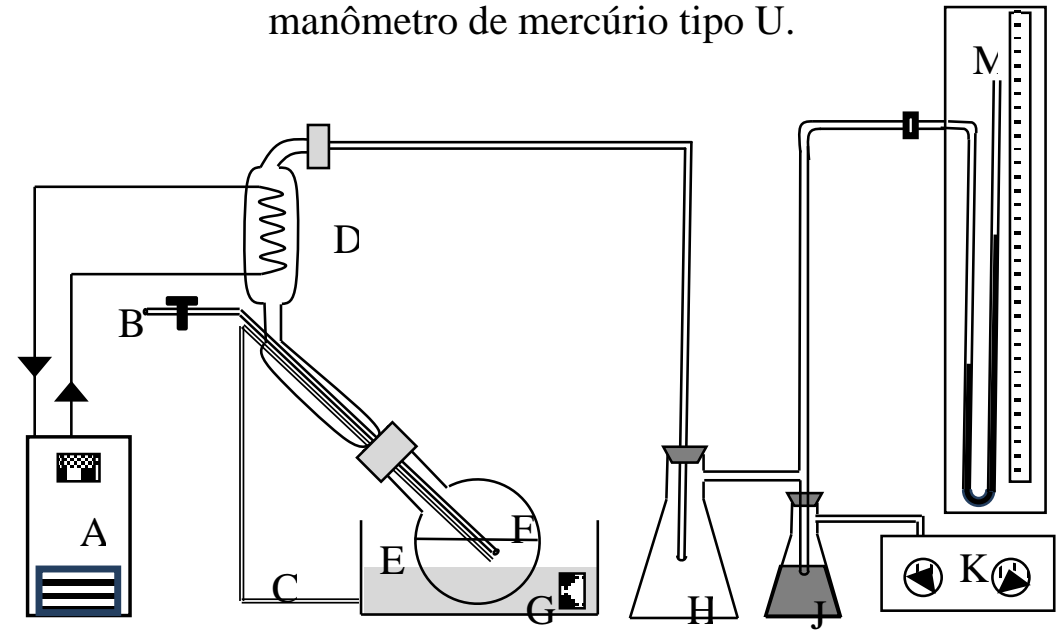




\section{RESULTADOS}

Os dados de pressão de vapor versus temperatura de ebulição das amostras 1,6 e 11 de leite/sacarose são apresentados na tabela 3 .

Tabela 3 - Valores experimentais de pressão de vapor pela temperatura de ebulição das amostras 1,6 e 11 .

\begin{tabular}{cccccc}
\hline \multicolumn{2}{c}{ Amostra 1 } & \multicolumn{2}{c}{ Amostra 6 } & \multicolumn{2}{c}{ Amostra 11 } \\
\hline Pv (KPa) & $\mathbf{T}(\mathbf{K})$ & $\mathbf{P v}(\mathbf{K P a})$ & $\mathbf{T}(\mathbf{K})$ & $\mathbf{P v}(\mathbf{K P a})$ & $\mathbf{T} \mathbf{( K )}$ \\
\hline 7,412 & 313,64 & 7,412 & 315,07 & 7,412 & 323,59 \\
7,572 & 314,05 & 7,812 & 316,05 & 7,505 & 323,82 \\
7,958 & 315,03 & 8,385 & 317,60 & 7,718 & 324,33 \\
10,611 & 320,64 & 8,598 & 317,94 & 9,118 & 328,16 \\
11,838 & 322,84 & 12,491 & 325,45 & 9,758 & 329,24 \\
13,184 & 325,03 & 12,598 & 325,59 & 10,331 & 330,44 \\
14,944 & 327,67 & 13,184 & 326,70 & 11,025 & 331,93 \\
16,277 & 329,42 & 13,851 & 327,59 & 11,371 & 332,60 \\
16,664 & 329,92 & 14,664 & 328,77 & 12,344 & 334,24 \\
16,677 & 329,93 & 17,304 & 332,26 & 14,238 & 337,28 \\
20,330 & 334,22 & 18,424 & 333,80 & 14,464 & 338,03 \\
26,902 & 340,40 & 18,997 & 334,28 & 19,797 & 344,74 \\
29,635 & 342,60 & 26,143 & 341,57 & 19,810 & 344,77 \\
29,729 & 342,66 & 27,769 & 342,94 & 22,823 & 347,97 \\
36,888 & 347,75 & 28,089 & 343,23 & 23,010 & 348,60 \\
37,954 & 348,39 & 29,262 & 343,97 & 25,116 & 350,30 \\
39,114 & 349,11 & 30,769 & 345,31 & 36,088 & 359,12 \\
39,247 & 349,18 & 39,234 & 350,86 & 42,727 & 363,32 \\
41,794 & 350,74 & 46,420 & 354,98 & 56,858 & 370,91 \\
49,219 & 354,70 & 50,299 & 357,20 & 62,978 & 373,82 \\
53,672 & 356,92 & 54,312 & 358,97 & 64,991 & 374,69 \\
70,150 & 363,83 & 55,938 & 359,72 & 67,524 & 375,60 \\
74,389 & 365,34 & 70,150 & 365,59 & 70,150 & 375,60 \\
\hline
\end{tabular}

Os dados das outras 8 amostras também se ajustaram bem ao modelo. A partir dessas 11 amostras de diferentes concentrações, e dos dados de pressão de vapor versus temperatura de evaporação de leite semidesnatado/sacarose, foram determinados os parâmetros da equação de Antoine (2) correspondente a cada uma das amostras de leite/sacarose.

Pressões de vapor de substancias puras como é o caso do leite podem ser aplicadas na equação de Antoine (2).

$$
\operatorname{Ln} \mathrm{Pv}=\mathrm{A}-[\mathrm{B} /(\mathrm{C}+\mathrm{T})]
$$

Foi então determinado o EPE de misturas leite/sacarose na faixa de temperatura de 40 a $100^{\circ} \mathrm{C}$ e concentração de sólidos totais entre 0,18072 e 0,70378 usando a equação empírica de Antoine (2) determinando os parâmetros dessa equação (A, B e C) em função da concentração, da pressão de vapor e consequentemente da temperatura de evaporação. Os parâmetros encontram-se listados na tabela 4 . 


\section{Congresso Brasileiro de Engenharia Química em Iniciação Científica UFSCar - São Carlos - SP 16 a 19 de Julho de 2017}

Tabela 4 - Parâmetros da equação (2) para leite semidesnatado/sacarose em diferentes concentrações de sólidos totais.

\begin{tabular}{ccccccc}
\hline $\begin{array}{c}\mathbf{N}^{\mathbf{0}} \text { de } \\
\text { Amostras }\end{array}$ & $\mathbf{X w}$ & $\mathbf{X s t}$ & $\mathbf{A}$ & $\mathbf{B}$ & $\mathbf{C}$ & $\mathbf{r}^{\mathbf{2}}$ \\
\hline 1 & 81,928 & 18,072 & 23,972 & 4303,598 & $-27,937$ & 0,999 \\
2 & 77,568 & 22,432 & 23,895 & 4260,805 & $-29,521$ & 0,999 \\
3 & 74,279 & 25,721 & 24,122 & 4405,784 & $-24,405$ & 0,999 \\
4 & 70,512 & 29,488 & 24,431 & 4609,315 & $-17,276$ & 0,999 \\
5 & 66,163 & 33,837 & 24,576 & 4712,036 & $-13,848$ & 0,999 \\
6 & 61,588 & 38,412 & 24,765 & 4847,501 & $-9,329$ & 0,999 \\
7 & 56,726 & 43,274 & 24,370 & 4610,341 & $-17,507$ & 0,999 \\
8 & 50,856 & 49,144 & 24,205 & 4518,695 & $-20,749$ & 0,999 \\
9 & 45,003 & 54,997 & 24,100 & 4497,486 & $-21,962$ & 0,999 \\
10 & 37,591 & 62,409 & 24,013 & 4500,573 & $-22,246$ & 0,999 \\
11 & 29,622 & 70,378 & 25,623 & 5680,735 & 16,294 & 0,999 \\
\hline
\end{tabular}

A tabela acima (tabela 4) indica uma boa correlação $\left(\mathrm{r}^{2}\right)$ entre o modelo utilizado e os dados experimentais das amostras de leite, podendo assim ser utilizados para a determinação do EPE de leite/sacarose.

\section{CONCLUSÃO}

Portanto, determinou-se os parâmetros da equação de Antoine para 11 amostras de leite/sacarose em pressões de vapor de 7,0 a 70,3KPa correlacionadas com a concentração de sólidos totais (de 0,1807 a 0,7037$)$ presente nas amostras a partir de experimentos da elevação do ponto de ebulição de leite/sacarose.

A análise dos dados relacionou bem o modelo de Antoine com os dados experimentais e assim os parâmetros dessa equação podem ser utilizados pela indústria para se determinar o EPE de soluções leite/sacarose em diferentes concentrações de sólidos totais, dado até então não disponível na literatura aberta e que podem proporcionar informações úteis para a modelagem, desenho e controle das operações unitárias envolvidas na indústria de produtos lácteos evaporados.

\section{NOMENCLATURA}

A, B e C: Constantes da equação de Antoine;

Pv: Pressão de Vapor (KPa);

$S_{y}$ : Desvio Padrão da amostra $(\mathrm{kJ} / \mathrm{kg} \cdot \mathrm{K})$,

$S_{y x}$ : Desvio padrão do modelo $(\mathrm{kJ} / \mathrm{kg} \cdot \mathrm{K})$;

T: Temperatura (K);

Xst: Concentração de sólidos totais (\%);

Xw: Concentração de água (\%) 


\section{REFERÊNCIAS}

CASTEll-PAlOU, A.; VÁQuiro, H. A.; CÁRCEL, J. A.; ROSSElló, C.; FEMENIA, A.; SIMAL, S. 2012. Mathematical Modeling of Moisture Distribution and Kinetics in Cheese Drying. Dry Ttchnol. V.30.

CONAB - Companhia Nacional de Abastecimento. Disponível em: <http://www.conab.gov.br/>. Acesso em: 20 de fevereiro de 2017.

IAL. INSTITUTO ADOLFO LUTZ. Normas Analíticas do Instituto Adolfo Lutz. Métodos químicos e físicos para análise de alimentos. $4^{\mathrm{a}}$ ed. São Paulo: Instituto Adolfo Lutz, 2008.

MINIM, A. Luis; COIMBRA, S. R. Jane; MINIM, P. R. Valéria; TELIS-ROMERO, Javier. Influence of Temperature and Water and Fat Contents on the Thermophysical Properties of Milk. Journal Of Chemical And Engineering Data. p. 1488-1491. 2002.

USDA - United States Departoneladaent of Agriculture. Disponível em: <https://www.usda.gov/>. Acesso em: 20 de fevereiro de 2017. 